\title{
Thermodynamic uncertainty relation in slowly driven quantum heat engines
}

\author{
Harry J. D. Miller, ${ }^{1}$ M. Hamed Mohammady, ${ }^{2}$ Martí Perarnau-Llobet, ${ }^{3}$ and Giacomo Guarnieri ${ }^{4,5}$ \\ ${ }^{1}$ Department of Physics and Astronomy, The University of Manchester, Manchester M13 9PL, UK. \\ ${ }^{2}$ RCQI, Institute of Physics, Slovak Academy of Sciences, Dúbravská cesta 9, Bratislava 84511, Slovakia \\ ${ }^{3}$ Département de Physique Appliquée, Université de Genève, Genève, Switzerland \\ ${ }^{4}$ School of Physics, Trinity College Dublin, College Green, Dublin 2, Ireland \\ ${ }^{5}$ Dahlem Center for Complex Quantum Systems, Freie Universität Berlin, 14195 Berlin, Germany
}

\begin{abstract}
Thermodynamic Uncertainty Relations express a trade-off between precision, defined as the noise-tosignal ratio of a generic current, and the amount of associated entropy production. These results have deep consequences for autonomous heat engines operating at steady-state, imposing an upper bound for their efficiency in terms of the power yield and its fluctuations. In the present manuscript we analyze a different class of heat engines, namely those which are operating in the periodic slow-driving regime. We show that an alternative TUR is satisfied, which is less restrictive than that of steady-state engines: it allows for engines that produce finite power, with small power fluctuations, to operate close to the Carnot efficiency. The bound further incorporates the effect of quantum fluctuations, which reduces engine efficiency relative to the average power and reliability. We finally illustrate our findings in the experimentally relevant model of a single-ion heat engine.
\end{abstract}

Introduction: Much like their macroscopic counterparts, microscopic heat engines function by converting a thermal energy current $J_{q}$ from their surrounding environment into power $P_{w} \geq 0$ [1-3]. In general, such engines can be divided into two classes: steady-state heat engines (SSHEs) and periodically driven heat engines (PDHEs). SSHEs are comprised of a working substance that is placed in weak contact with multiple reservoirs, so that the ensuing Markovian dynamics results in the engine reaching a non-equilibrium steady-state in the long time limit, thereby supporting a net constant power current [4]. On the other hand, PDHEs are operated by periodically changing both the mechanical parameters of the working substance, as well as the temperature of its surrounding reservoir, thus generating power by external driving $[5,6]$. In both cases, for any engine operating between a hot and cold temperature, $T_{h}>T_{c}$, standard thermodynamic laws ensure that the efficiency $\eta:=P_{w} / J_{q}$ cannot exceed Carnot's bound: $\eta \leq \eta_{C}:=1-\left(T_{c} / T_{h}\right)$. In addition to this, microscopic engines are significantly influenced by stochastic fluctuations, which can be of thermal or quantum origin. Understanding how these fluctuations impact the performance of small-scale machines is a central goal of both classical-stochastic [7], and quantum [8, 9], thermodynamics, as they determine the engine's reliability.

Recently, Pietzonka and Seifert found that the efficiency of SSHEs is constrained by a bound tighter than Carnot [10]:

$$
\eta \leq \frac{\eta_{C}}{1+2 T_{c} P_{w} / \Delta P_{w}}=: \eta^{P S} .
$$

This bound incorporates an additional dependence on the engine's time-averaged work fluctuations $\Delta P_{w}$. The quantity $\Delta P_{w}$ represents the so-called constancy of the engine [10], which inversely quantifies the engine's reliability in terms of power output. The bound Eq. (1) tells us that in order to increase the efficiency of any SSHE, one must either sacrifice the power output $P_{w}$ or the engine's reliability. This can be seen as a consequence of the thermodynamic uncertainty relation (TUR) [11-13], which states that entropy production constrains the noise-to-signal ratio of any current in SSHEs. Extensions and generalizations of Eq. (1) to autonomous quantum systems operating at steady-state have been investigated [14-18].

With regard to PDHEs, it is still currently debated whether a similar universal trade-off is expected to hold: on the one hand, it was found that both in the case of an externally driven Brownian clock [19] and in driven cyclic heat engines [20] one can achieve small fluctuations at finite power output in a dissipationless manner. On the other hand, TUR-bounds for driven Langevin systems [21] and dissipative two-level systems [22], as well as for classical timedependent driven engines [23] were found. In general, TURs giving rise to Eq. (1) can be recovered for protocols that are time-symmetric [24], or modified in order to account for time-asymmetry in the small-amplitude regime [15]. Alternatively, other bounds have also been derived with an additional dependence on hysteresis $[18,25]$ or driving frequency [26]. However, in all above cases a general quantum mechanical trade-off between efficiency, average power and its variance has not yet been achieved. Moreover, the impact of quantum fluctuations on such a trade-off has yet to be established. In this paper, we provide these important missing pieces of the puzzle, by deriving the following quantum version of Eq. (1) for PDHEs operating in the slow driving, Markovian regime:

$$
\eta \leq \frac{\eta_{C}}{1+2 T_{c} P_{w} f\left(\left|\frac{P_{\mathcal{W}}}{P_{w}}\right|\right) /\left(\Delta P_{w}-2 \Delta \mathcal{I}_{w}\right)}:=\eta^{Q},
$$

where: $P_{\mathcal{W}}$ denotes the adiabatic (also known as quasistatic [27]) power; $f(x):=(1-x)^{2}$; and $0 \leq 2 \Delta \mathcal{I}_{w} \leq \Delta P_{w}$ is a quantum correction term, which will be precisely defined below. Firstly, we see that Eq. (2) is structurally different from Eq. (1) as it now depends on the ratio between actual and adiabatic power. Depending on this ratio, the bound may exceed or fall below the SSHE bound Eq. (1). Furthermore, the term $\Delta \mathcal{I}_{w}$ represents a measure of quantum fluctuations of the power as it depends purely on quantum friction [28-31], and has recently been shown to lead to a 
quantum correction to the standard fluctuation-dissipation relation for work $[32,33]$. Crucially, $\eta^{Q}$ is a decreasing function with respect to $\Delta \mathcal{I}_{w}$, meaning that quantum fluctuations have a negative impact on the performance of PDHEs in the slow driving regime, making it impossible to achieve the optimal classical efficiency for a given average power output and its variance.

Periodic quantum heat engines: We consider engines where the working medium is a driven quantum system, weakly coupled to a heat bath. Setting $\hbar=k_{B}=1$, this is described by a time-dependent adiabatic Lindblad master equation of the form $\dot{\rho}_{t}=\mathscr{L}_{\lambda(t)}\left(\rho_{t}\right)$ [34], where the time-dependence exhibited by the dynamical generator $\mathscr{L}_{\lambda(t)}$ is protocol-dependent, and is induced by the external modulation of the bath temperature $T(t)$ and control mechanical parameters $\Lambda(t)$ which determine the Hamiltonian $H_{\Lambda(t)}$. An engine cycle of duration $\tau$ is then represented by a closed curve in the control parameters space $\lambda: t \mapsto \lambda(t):=\{T(t), \Lambda(t)\}$, such that it satisfies $\lambda(0)=\lambda(\tau)$. In particular, following [5, 35], we parameterise the temperature modulation as

$$
T(t):=\frac{T_{c} T_{h}}{T_{h}+\left(T_{c}-T_{h}\right) \alpha(t)}, \quad T_{c} \leq T_{h},
$$

with $\alpha(t) \in[0,1]$, and $\alpha(0)=\alpha(\tau)=0$. This implies that at $t=0, \tau$, the thermal bath that is in contact with the system is at the cold temperature $T_{c}$, but approaches the hot temperature $T_{h}$ in the middle of the cycle. From now on we further assume that, for all $t$, the quantum detailed balance condition $[36,37]$ is satisfied, and that there exists a unique stationary state $\pi_{\lambda(t)}$, such that $\mathscr{L}_{\lambda(t)}\left(\pi_{\lambda(t)}\right)=0$, which is of Gibbs form. This means that $\pi_{\lambda(t)}=e^{-\beta(t) H_{\Lambda(t)}} / Z_{\lambda(t)}$, where $\beta(t):=1 / T(t)$ is the inverse temperature and $Z_{\lambda(t)}:=\operatorname{Tr}\left(e^{-\beta(t) H_{\Lambda(t)}}\right)$ is the partition function.

A central quantity of interest throughout our analysis is the non-adiabatic entropy production rate, defined as

$$
\langle\dot{\sigma}\rangle:=\frac{\langle\sigma\rangle}{\tau}=\frac{1}{\tau}\left(\Delta S-\int_{0}^{\tau} d t \beta(t)\langle\dot{q}(t)\rangle\right) \geq 0,
$$

where $\langle\dot{q}(t)\rangle:=\operatorname{Tr}\left(\dot{\rho}_{t} H_{\Lambda(t)}\right)$ is (in weak coupling) the rate of heat entering the system and $\Delta S$ the increase in information entropy. Eq. (4) quantifies the dissipation in terms of excess heat in order to drive a system out of equilibrium, and can be directly related to the degree of irreversibility of a process [38,39]. Using Eq. (3) and the periodic boundary conditions, one can easily show that Eq. (4) takes the form

$$
\langle\dot{\sigma}\rangle=\frac{1}{T_{c}}\left(\eta_{C} J_{q}-P_{w}\right) \geq 0,
$$

where we have introduced the time-average power and heat flux supplied to the engine [5]:

$$
\begin{aligned}
P_{w} & :=-\frac{1}{\tau} \int_{0}^{\tau} d t \operatorname{Tr}\left(\dot{H}_{\Lambda(t)} \rho_{t}\right), \\
J_{q} & :=\frac{1}{\tau} \int_{0}^{\tau} d t \alpha(t) \operatorname{Tr}\left(H_{\Lambda(t)} \dot{\rho}_{t}\right) .
\end{aligned}
$$

Naturally, this decomposition leads us to define the efficiency as the ratio $\eta:=P_{w} / J_{q}$ between power output and heat flux entering the machine, which for an engine (defined by the regime $P_{w} \geq 0$ ) is bounded by the Carnot efficiency $\eta \leq \eta_{C}:=1-T_{c} / T_{h}$ due to the second law Eq. (4). We note that $\alpha(t)$ plays the role of a weighting function for the heat flux Eq. (7), with increasing weight assigned to increasing temperatures. This generalises the traditional thermodynamic efficiency where the system interacts with only two baths at distinct temperatures, which is recovered by choosing $\alpha(t)$ to be a step function. In this case, it is easy to see that $J_{q}$ reduces to the heat flow from the hot bath and the standard definition of efficiency is recovered [5].

In this paper we are finally concerned with engines that operate in the slow-driving regime, which are characterised by choosing the driving protocol $\lambda(t)$ as a slowly varying periodic function, satisfying boundary conditions $\dot{\lambda}(0)=$ $\dot{\lambda}(\tau)=\{0,0\}$. This ensures that the system occupies the same equilibrium state $\pi_{\lambda(0)}$ at the start and end of the cycle, and remains close to the instantaneous steady-state at all times during the cycle, taking the form $\rho_{t} \simeq \pi_{\lambda(t)}+$ $\delta \rho_{t}$, where $\delta \rho_{t}$ is a traceless correction term that vanishes linearly with the driving speed $[32,40,41]$. This regime is physically reached by setting the engine cycle duration $\tau$ to be large relative to the intrinsic relaxation timescale $t^{e q}$ of the system [42]. In order to evaluate the leading order terms of Eqs. (5) and (6) in the slow-driving regime, let us first define a self-adjoint operator-valued function $\delta A_{\lambda}:[0, \tau] \ni$ $t \mapsto \delta A_{\lambda(t)}$, where $A_{\lambda(t)}$ can be any self-adjoint operator, and $\delta: A \mapsto \delta A:=A-\operatorname{Tr}\left(A \pi_{\lambda(t)}\right) \mathbb{I}$ is a "spectrum shift" that ensures $\operatorname{Tr}\left(\delta A_{\lambda(t)} \pi_{\lambda(t)}\right)=0$ for all $A_{\lambda(t)}$. In Appendix $A$, we show that under the assumption of detailed balance and uniqueness of the instantaneous steady state $\left(\mathscr{L}_{\lambda(t)}\left(\pi_{\lambda(t)}\right)=0\right)$, the following is a valid inner product for such operator-valued functions:

$$
\begin{aligned}
& \left\langle\left\langle\delta A_{\lambda}, \delta B_{\lambda}\right\rangle\right\rangle_{\lambda}:=\frac{1}{2 \tau} \int_{0}^{\tau} d t \int_{0}^{\infty} d \theta \\
& \operatorname{Tr}\left(\delta A_{\lambda(t)}(\theta) \mathbb{J}_{\lambda(t)}\left(\delta B_{\lambda(t)}\right)+\delta B_{\lambda(t)}(\theta) \mathbb{J}_{\lambda(t)}\left(\delta A_{\lambda(t)}\right)\right),
\end{aligned}
$$

where $\delta A_{\lambda(t)}(\theta):=e^{\theta \mathscr{L}_{\lambda(t)}^{*}}\left(\delta A_{\lambda(t)}\right)$, with $\mathscr{L}_{\lambda(t)}^{*}$ the generator in the Heisenberg picture, and $\mathbb{J}_{\lambda(t)}(\cdot):=$ $\int_{0}^{1} d s \pi_{\lambda(t)}^{s}(\cdot) \pi_{\lambda(t)}^{1-s}$. The results of $[32,40,41,43,44]$ provide a means of Taylor expanding Eqs. (5) and (6) up to first order in $t^{e q} / \tau$, which can be shown in terms of the inner product Eq. (8) as

$$
\langle\dot{\sigma}\rangle=\left\langle\left\langle\delta \dot{X}_{\lambda}, \delta \dot{X}_{\lambda}\right\rangle\right\rangle_{\lambda}, \quad P_{w}=P_{\mathcal{W}}-\left\langle\left\langle\delta \dot{H}_{\lambda}, \delta \dot{X}_{\lambda}\right\rangle\right\rangle_{\lambda},
$$

where $X_{\lambda(t)}:=\beta(t) H_{\Lambda(t)}$, while $\delta \dot{H}_{\lambda(t)} \equiv \delta \dot{H}_{\Lambda(t)}$. Moreover, we have introduced the adiabatic power as

$$
P_{\mathcal{W}}:=-\frac{1}{\tau} \int_{0}^{\tau} d t \operatorname{Tr}\left(\dot{H}_{\Lambda(t)} \pi_{\lambda(t)}\right),
$$


which is the engine's power assuming that the system is in equilibrium at all times (compare with Eq. (6)), achieved in the limit $t^{e q} / \tau \rightarrow 0$.

So far we have only considered ensemble averages of thermodynamic quantities. For quantum-mechanical systems, the higher order statistics associated with work become preponderant and fundamentally depend on the measurement scheme used to monitor the system. In the case of open quantum systems whose dynamics are described by a timedependent Lindblad master equation, the fluctuating work can be determined at the stochastic level by monitoring sequences of quantum jumps exhibited by the system as it interacts with an environment [45-54]. Each time a jump occurs heat is exchanged between engine and environment, and this can be experimentally monitored through an external quantum detector [55-57]. Alternatively, one may determine the fluctuating work from two-time global energy measurements, on both the system and bath, at the beginning and end of the cycle $[58,59]$. In the Markovian limit with weak coupling between system and bath, both approaches allow one to arrive at a general expression for the time-averaged work variance, dependent only on the system degrees of freedom [32,60], which takes the following form in the slow driving limit:

$$
\Delta P_{w}=2\left(\Delta \mathcal{I}_{w}+\left\langle\left\langle\delta \dot{H}_{\lambda}, \delta \dot{H}_{\lambda}\right\rangle\right\rangle_{\lambda}\right) .
$$

Here, we have identified a quantum correction term due to the fluctuations,

$$
\Delta \mathcal{I}_{w}:=\frac{1}{\tau} \int_{0}^{\tau} d t \tau_{\lambda(t)}^{e q} \mathcal{I}_{\lambda(t)}\left(\dot{H}_{\Lambda(t)}, \dot{H}_{\Lambda(t)}\right),
$$

where we introduce the skew covariance [61, 62]

$$
\mathcal{I}_{\lambda(t)}(A, B):=-\frac{1}{2} \int_{0}^{1} d s \operatorname{Tr}\left(\left[A, \pi_{\lambda(t)}^{s}\right]\left[B, \pi_{\lambda(t)}^{1-s}\right]\right) .
$$

The skew information $\mathcal{I}_{\lambda(t)}(A, A) \geq 0$ represents a measure of quantum fluctuations in the sharp observable $A=A^{\dagger}$ with respect to instantaneous equilibrium $\pi_{\lambda(t)}$ [63-67]. In particular, the skew information vanishes for $\left[A, \pi_{\lambda(t)}\right]=0$, reduces to the usual variance $\left\langle A^{2}\right\rangle-\langle A\rangle^{2}$ for pure states, and is convex under classical mixing. In this context, $\mathcal{I}_{\lambda(t)}\left(\dot{H}_{\Lambda(t)}, \dot{H}_{\Lambda(t)}\right)$ measures the degree of quantum power fluctuations due to the generation of quantum friction stemming from $\left[\dot{H}_{\Lambda(t)}, H_{\Lambda(t)}\right] \neq 0[28-33]$. Additionally, these quantum fluctuations are weighted by an integral relaxation timescale:

$$
\tau_{\lambda(t)}^{e q}:=\int_{0}^{\infty} d \theta \frac{\mathcal{I}_{\lambda(t)}\left(\dot{H}_{\Lambda(t)}(\theta), \dot{H}_{\Lambda(t)}(0)\right)}{\mathcal{I}_{\lambda(t)}\left(\dot{H}_{\Lambda(t)}(0), \dot{H}_{\Lambda(t)}(0)\right)} \geq 0 .
$$

This quantifies the timescale over which the quantum correlation function for the power decays to its equilibrium value, and can be viewed as a quantum generalisation of the integral relaxation time employed in classical non-equilibrium thermodynamics $[27,68,69]$.
Quantum bound on efficiency. We are now ready to derive bounds on the performance of quantum PDHEs. By noting that $\langle\dot{\sigma}\rangle, P_{w}$, and $\left(\Delta P_{w}-2 \Delta \mathcal{I}_{w}\right)$ can all be expressed through the inner product introduced in Eq. (8) we can apply the Cauchy-Schwarz inequality $\left\langle\left\langle A_{\lambda}, A_{\lambda}\right\rangle\right\rangle_{\lambda}\left\langle\left\langle B_{\lambda}, B_{\lambda}\right\rangle\right\rangle_{\lambda} \geq\left|\left\langle\left\langle A_{\lambda}, B_{\lambda}\right\rangle\right\rangle_{\lambda}\right|^{2}$, thus obtaining our central result:

$$
\left(\Delta P_{w}-2 \Delta \mathcal{I}_{w}\right)\langle\dot{\sigma}\rangle \geq 2 f\left(\left|\frac{P_{\mathcal{W}}}{P_{w}}\right|\right) P_{w}^{2}
$$

where $f(x):=(1-x)^{2}$. The above inequality is a quantum generalisation of the TUR for entropy production and power in PDHEs. It demonstrates a trade-off between the entropy production rate $\langle\dot{\sigma}\rangle$ and the noise-to-signal ratio of power, $\sqrt{\Delta P_{w}} / P_{w}$. This TUR has an immediate consequence for the achievable engine efficiency: a simple rewriting of Eq. (5) as $\eta=\eta_{C} P_{w} /\left(T_{c}\langle\dot{\sigma}\rangle+P_{w}\right)$, and combination with Eq. (15) produces the desired efficiency bound Eq. (2).

When comparing Eq. (2) to the TUR bound for SSHEs Eq. (1), we notice a modification stemming both from the ratio between adiabatic and actual power $\left|P_{\mathcal{W}} / P_{w}\right|$, as well as from the presence of $\Delta \mathcal{I}_{w}$. We show in Appendix $A$ that this quantum correction satisfies $0 \leq 2 \Delta \mathcal{I}_{w} \leq \Delta P_{w}$, which means that $\eta^{Q}$ decreases monotonically with increasing $\Delta \mathcal{I}_{w}$. As a consequence, the bound Eq. (2) is in fact more restrictive than the equivalent classical engine bound with vanishing quantum fluctuations, namely $\eta^{Q} \leq \eta^{\mathrm{cl}}:=$ $\eta^{Q}$ s.t. $\Delta \mathcal{I}_{w}=0$. This demonstrates the detrimental influence of quantum friction for PDHEs close to equilibrium. Indeed, to saturate Eq. (2) we require that, for all times $t \in[0, \tau], \delta \dot{H}_{\lambda(t)} \propto \delta \dot{X}_{\lambda(t)}$. However, this necessarily implies a vanishing quantum friction $\left[\dot{H}_{\Lambda(t)}, H_{\Lambda(t)}\right]=0$ for all $t \in[0, \tau]$, which is a necessary and sufficient condition for $\Delta \mathcal{I}_{w}=0$. Hence, Eq. (2) is in fact a strict inequality for heat engines in the presence of quantum friction. As expected and discussed in Refs. [32, 33], the quantum correction becomes most relevant at low temperatures, which we will later illustrate by an example.

The correction arising from $\left|P_{\mathcal{W}} / P_{w}\right|$ affects engines irrespectively of quantum friction, and can lead to large deviations from Eq. (1). First, we note that for time-symmetric protocols, where $P_{\mathcal{W}}=0$, we recover the original bound, which is in agreement with [24]. Moving slightly away from this point, one may find a regime where $P_{\mathcal{W}}>2 P_{w}$. In this case, $f\left(\left|P_{\mathcal{W}} / P_{w}\right|\right)>1$, and the bound Eq. (2) becomes more restrictive than that of SSHEs Eq. (1). However, in most operating regimes with non-vanishing power output we expect $f\left(\left|P_{\mathcal{W}} / P_{w}\right|\right) \ll 1$, as $P_{\mathcal{W}}-P_{w}=\mathcal{O}\left(t^{e q} / \tau\right) \ll 1$ in the slow driving regime, see the expansion Eq. (9) (recall that $t^{e q}$ is the characteristic equilibration timescale). That is, the power-efficiency of slowly driven PDHEs is less constrained by fluctuations than that of SSHEs. To quantify this, one may expand Eq. (2) in $\epsilon \equiv t^{e q} / \tau$ obtaining:

$$
\eta \leq \eta_{C}\left(1-\epsilon \frac{2 T_{c} a_{P}^{2}}{P_{\mathcal{W}} a_{\Delta P}}\right)+\mathcal{O}\left(\epsilon^{2}\right),
$$

where we have defined $a_{P} \equiv \lim _{\epsilon \rightarrow 0}\left(P_{w}-P_{\mathcal{W}}\right) / \epsilon$ and 

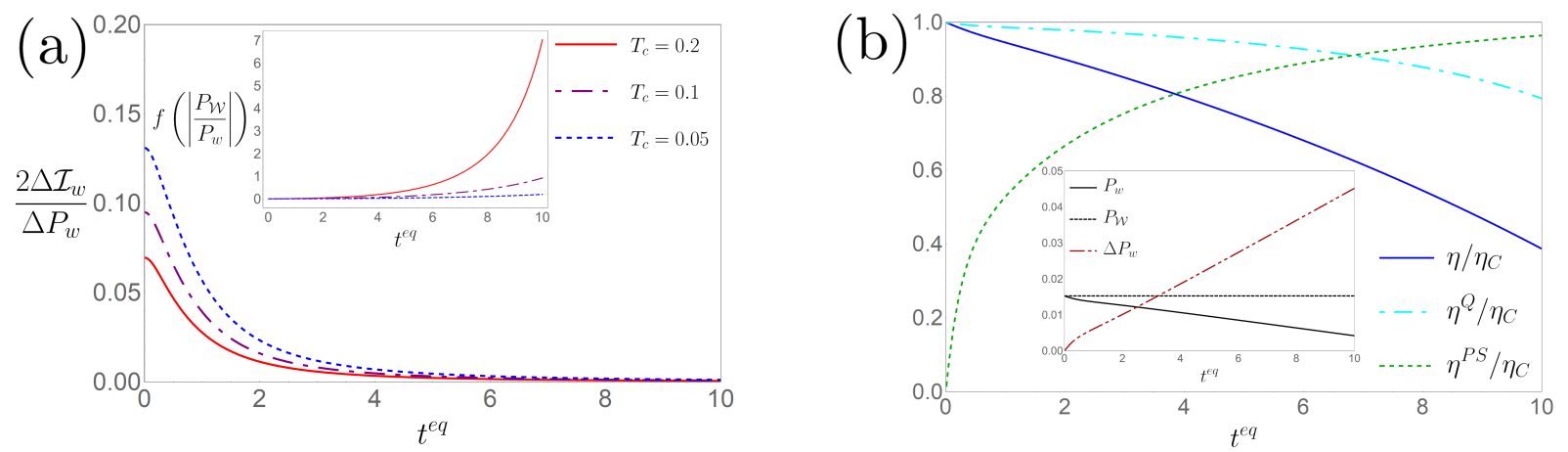

FIG. 1. Here, we simulate the single ion engine, defined by the protocol in Eq. (18), with the parameters $\omega_{0}=1, T_{h}=2$, and $\tau=100$, where we choose units of $\hbar=k_{B}=1$. (a) Plot of the ratio between quantum and total power fluctuations, $2 \Delta \mathcal{I}_{w} / \Delta P_{w}$, as a function of $t^{e q}:=1 / \Gamma$ and $T_{c}$. In the inset, we evaluate the correction term for the bound Eq. (2) due to the ratio between the adiabatic and actual power. (b) Plot of the efficiency $\eta$ and the bounds $\eta^{P S}$, Eq. (1), and $\eta^{Q}$, Eq. (2), as a function of $t^{e q}:=1 / \Gamma$, for $T_{c}=0.2$. In the inset, we evaluate the power $P_{w}$, adiabatic power $P_{\mathcal{W}}$, and power fluctuations $\Delta P_{w}$.

$a_{\Delta P} \equiv \lim _{\epsilon \rightarrow 0}\left(\Delta P_{w}-2 \Delta \mathcal{I}_{w}\right) / \epsilon$, which are finite and can be inferred from Eqs. (9) and (11).

Since the bound can be saturated for $\delta \dot{H}_{\lambda} \propto \delta \dot{X}_{\lambda}$, one can in principle approach the Carnot efficiency, at finite power and zero work fluctuations, in the limit where $t^{e q}$ becomes vanishingly small and $\tau$ remains finite (recall that work fluctuations are proportional to $\epsilon$ from Eq. (11)). This is in accordance with the results demonstrated in [20,70]. Our bound Eq. (2) thus incorporates previous results and furthermore gives the leading order correction to Carnot's bound for finite speed and equilibration time-scale, while clarifying that PDHEs also obey TUR relations.

Single ion heat engine: To illustrate our bound Eq. (2) we consider a model of a single ion PDHE, inspired by recent experimental realisations using ion-traps [71]. We describe the engine using a master equation for the damped harmonic oscillator:

$$
\dot{\rho}_{t}=-i \omega\left[a_{\omega}^{\dagger} a_{\omega}, \rho_{t}\right]+\Gamma\left(N_{\beta}+1\right) \mathcal{D}_{a_{\omega}}\left[\rho_{t}\right]+\Gamma \mathcal{D}_{a_{\omega}^{\dagger}}\left[\rho_{t}\right],
$$

with $\mathcal{D}_{X}[\rho]=X \rho X^{\dagger}-\frac{1}{2}\left\{X^{\dagger} X, \rho\right\}$. Here the Hamiltonian is $H_{\omega}=\omega\left(a_{\omega}^{\dagger} a_{\omega}+\frac{1}{2}\right)$ with $\omega$ the time-dependent frequency, $a_{\omega}=\sqrt{\omega / 2}(x+i p / \omega)$ is the creation operator with unit mass, $\Gamma$ is the damping rate (in the slow driving regime, $t_{\mathrm{eq}} / \tau \ll 1$ with $\left.t_{\mathrm{eq}} \equiv \Gamma^{-1}\right)$, and $N_{\beta}=1 /\left(e^{\beta \omega}-1\right)$ is the Bose-Einstein distribution. We consider a cycle defined by the slow modulation of the engine's oscillator frequency and bath temperature, $\lambda: t \mapsto \lambda(t)=\{T(t), \omega(t)\}$, according to the periodic functions

$$
\begin{aligned}
& \omega(t)=\omega_{0}\left(1+\frac{1}{2} \sin \left(\frac{2 \pi t}{\tau}\right)+\frac{1}{4} \sin \left(\frac{4 \pi t}{\tau}+\pi\right)\right), \\
& T(t)=\frac{T_{c} T_{h}}{T_{h}+\left(T_{c}-T_{h}\right) \sin ^{2}\left(\frac{\pi t}{\tau}\right)}
\end{aligned}
$$

where $T_{c}<T_{h}$ and $\omega_{0}>0$. Note that for the temperature, we have simply assigned $\alpha(t)=\sin ^{2}(\pi t / \tau)$ in Eq. (3). This protocol is cyclic, $\omega(0)=\omega(\tau)=\omega_{0}, T(0)=T(\tau)=T_{c}$, and satisfies the slow-driving condition $\dot{\omega}(t)=\dot{T}(t)=0$ for $t=0, \tau$. In Appendix B we calculate the power and its fluctuations, as well as the efficiency and its bounds, using Eqs. (5), (9), and (11). Notably, the power operator $\dot{H}_{\omega}=\dot{\omega}\left(\omega^{-1} H_{\omega}+\left(\left(a_{\omega}^{\dagger}\right)^{2}+a_{\omega}^{2}\right) / 2\right)$ does not commute with the engine Hamiltonian, $\left[H_{\omega}, \dot{H}_{\omega}\right] \neq 0$, meaning that quantum friction is present throughout the cycle, and so the quantum correction term Eq. (12) is strictly positive. To see this effect, we plot the ratio between quantum and total power fluctuations, i.e. $2 \Delta \mathcal{I}_{w} / \Delta P_{w}$, in Fig. 1 (a). It can be seen how the quantum fluctuations become more relevant in the low temperature regime, $\omega_{0} / T_{c} \gg 1$, as expected. In this regime, the TUR Eq. (15) might become substantially affected by quantum fluctuations. Moreover, while the total power fluctuations $\Delta P_{w}$ vanish as $t^{e q} / \tau \rightarrow 0$ (see the inset of Fig. 1 (b)), the ratio of the quantum fluctuations $2 \Delta \mathcal{I}_{w} / \Delta P_{w}$ can be seen to increase as $t^{e q} / \tau$ becomes smaller, showing that quantum fluctuations becomes more relevant in the slow-driving limit. Conversely, the correction term $f\left(\left|P_{\mathcal{W}} / P_{w}\right|\right)$ is large when $t^{e q} / \tau$ is large, and vanishes in the limit $t^{e q} / \tau \rightarrow 0$. This means that, as can be seen in Fig. 1 (b), in the limit $t^{e q} / \tau \rightarrow 0$ the engine produces finite power $P_{w}=P_{\mathcal{W}}$, while both the efficiency $\eta$, as well as the bound given in Eq. (2), approaches Carnot. Finally, we see in Fig. 1 (b) that the bound in Eq. (1) does not apply to PDHEs; while the efficiency $\eta$ always obeys Eq. (2), it can violate Eq. (1) for sufficiently small $t^{e q} / \tau$, as this bound vanishes in the slow-driving limit, due to the fact that $\Delta P_{w}$ becomes vanishingly small.

Conclusions: We have derived a bound on the optimal efficiency of quantum periodically driven heat engines (PDHEs) in terms of their average power and constancy, valid in the slow-driving, Markovian regime. In the first instance, we see that PDHEs are subject to a bound that differs from steady-state heat engines (SSHEs) through an additional dependence on the ratio between adiabatic and actual power. Nonetheless, Eq. (2) still imposes a universal constraint on engine efficiency for a given power and constancy, thus providing a finite time correction to the Carnot bound at leading order in driving speed. The bound further incorporates the effect of quantum friction stemming 
from possibly non-commuting Hamiltonian driving. This represents the first Thermodynamic Uncertainty Relation for PDHEs that explicitly shows the role of quantum effects.

It has recently been shown that quantum friction reduces the maximum power achievable in slow driving PDHEs [41, $44,72,73]$. Our results demonstrate that in this operational regime, quantum friction limits the efficiency relative to the subsequent reliability and power. More specifically, when optimising any one of the trio $\phi \in\left\{\eta, P_{w}, 1 / \Delta P_{w}\right\}$ while fixing the other two variables, quantum friction inevitably leads to a reduction in the maximum value $\phi_{\max }$ that can be attained. Given that enhancements with a quantum origin have been identified in other thermodynamic contexts, such as Otto-like engines [74-76] or refrigerators [77], a full understanding of the role of quantum effects in PDHEs beyond the slow-driving and weak-coupling regime remain as open questions.

\section{Acknowledgments}

H. J. D. M. acknowledges support from the Royal Commission for the Exhibition of 1851. M. H. M. acknowledges support from the Slovak Academy of Sciences under MoRePro project OPEQ (19MRP0027), as well as projects OPTIQUTE (APVV-18-0518) and HOQIT (VEGA 2/0161/19). M. P.-L. acknowledges funding from Swiss National Science Foundation (Ambizione PZ00P2-186067). G. G. acknowledges support from the European Research Council Starting Grant ODYSSEY (G. A. 758403).
[1] V. Blickle and C. Bechinger, Nat. Phys. 8, 143 (2012).

[2] J. P. Pekola, Nat. Phys. 11, 118 (2015).

[3] A. Ronzani, B. Karimi, J. Senior, Y.-C. Chang, J. T. Peltonen, C. Chen, and J. P. Pekola, Nat. Phys. 14, 991 (2018).

[4] G. Benenti, G. Casati, K. Saito, and R. S. Whitney, Phys. Rep. 1, 694 (2017).

[5] K. Brandner and U. Seifert, Phys. Rev. E 93, 062134 (2016).

[6] M. H. Mohammady and A. Romito, Phys. Rev. E 100, 012122 (2019).

[7] U. Seifert, Rep. Prog. Phys 75, 126001 (2012).

[8] M. Campisi, P. Hänggi, and P. Talkner, Rev. Mod. Phys, 83, 771 (2011).

[9] J. Goold, M. Huber, A. Riera, L. del Rio, and P. Skrzypczyk, J. Phys. A 49, 143001 (2016).

[10] P. Pietzonka and U. Seifert, Phys. Rev. Lett. 120, 190602 (2018).

[11] T. R. Gingrich, J. M. Horowitz, N. Perunov, and J. L. England, Phys. Rev. Lett. 116, 120601 (2016).

[12] A. C. Barato and U. Seifert, Phys. Rev. Lett. 114, 158101 (2015).

[13] J. M. Horowitz and T. R. Gingrich, Nat. Phys. 16, 15 (2020).

[14] G. Guarnieri, G. T. Landi, S. R. Clark, and J. Goold, Phys. Rev. Research 1, 033021 (2019).

[15] K. Macieszczak, K. Brandner, and J. P. Garrahan, Phys. Rev. Lett. 121, 130601 (2018).

[16] Y. Hasegawa, Phys. Rev. Lett. 125, 050601 (2020).

[17] Y. Hasegawa, Phys. Rev. Lett. 126, 010602 (2021).

[18] P. P. Potts and P. Samuelsson, Phys. Rev. E 100, 052137 (2019).

[19] A. C. Barato and U. Seifert, Phys. Rev. X 6, 041053 (2016).

[20] V. Holubec and A. Ryabov, Phys. Rev. Lett. 121, 120601 (2018).

[21] T. Van Vu and Y. Hasegawa, Phys. Rev. Research 2, 013060 (2020).

[22] L. M. Cangemi, V. Cataudella, G. Benenti, M. Sassetti, and G. D. Filippis, Phys. Rev. B 102, 165418 (2020).

[23] T. Koyuk and U. Seifert, Phys. Rev. Lett. 125, 260604 (2020).

[24] K. Proesmans and C. V. D. Broeck, Euro. Phys Lett. 119, 20001 (2017).

[25] K. Proesmans and J. M. Horowitz, J. Stat. Mech. 2019,
054005 (2019).

[26] T. Koyuk and U. Seifert, Phys. Rev. Lett. 122, 230601 (2019).

[27] D. Mandal and C. Jarzynski, J. Stat. Mech. 2016, 063204 (2016).

[28] T. Feldmann and R. Kosloff, Phys. Rev. E 68, 016101 (2003).

[29] F. Plastina, A. Alecce, T. J. G. Apollaro, G. Falcone, G. Francica, F. Galve, N. L. Gullo, and R. Zambrini, Phys. Rev. Lett. 113, 260601 (2014).

[30] G. Francica, J. Goold, and F. Plastina, Phys. Rev. E 99, 042105 (2019).

[31] R. Dann and R. Kosloff, N. J. Phys 22, 013055 (2020).

[32] H. J. D. Miller, M. Scandi, J. Anders, and M. PerarnauLlobet, Phys. Rev. Lett. 123, 230603 (2019).

[33] M. Scandi, H. J. D. Miller, J. Anders, and M. PerarnauLlobet, Phys. Rev. Research 2, 023377 (2020).

[34] T. Albash, S. Boixo, and D. A. Lidar, N. J. Phys 14, 123016 (2012).

[35] K. Brandner, K. Saito, and U. Seifert, Phys. Rev. X 5, 031019 (2015).

[36] R. Alicki and K. Lendi, Quantum Dynamical Semigroups and Applications (Springer, 1987).

[37] R. Alicki, Rep. Math. Phys. 10, 249 (1976).

[38] M. Esposito and C. Van Den Broeck, Phys. Rev. Lett. 104, 090601 (2010).

[39] M. Esposito and C. Van den Broeck, Phys. Rev. E 82, 011143 (2010).

[40] V. Cavina, A. Mari, and V. Giovannetti, Phys. Rev. Lett. 119, 050601 (2017).

[41] K. Brandner and K. Saito, Phys. Rev. Lett. 124, 040602 (2020).

[42] The intrinsic relaxation timescale $t^{e q}$ of the system is determined by the inverse spectral gap of the Lindbladian.

[43] M. Scandi and M. Perarnau-Llobet, Quantum 3, 197 (2019).

[44] P. Abiuso and M. Perarnau-Llobet, Phys. Rev. Lett. 124, 110606 (2020).

[45] J. M. Horowitz, Phys. Rev. E 85, 1 (2012).

[46] J. M. Horowitz and J. M. R. Parrondo, N. J. Phys 15, 085028 (2013).

[47] J. M. Horowitz and T. Sagawa, J. Stat. Phys. 156, 55 (2014). 
[48] G. Manzano, J. M. Horowitz, and J. M. R. Parrondo, Phys. Rev. E 92, 032129 (2015).

[49] F. Liu, Phys. Rev. E 93, 012127 (2016).

[50] F. Liu and J. Xi, Phys. Rev. E 94, 062133 (2016).

[51] G. Manzano, J. M. Horowitz, and J. M. R. Parrondo, Phys. Rev. X 8, 31037 (2018).

[52] C. Elouard and M. H. Mohammady, in Thermodynamics in the quantum regime: Fundamental Aspects and New Directions, Fundamental Theories of Physics, Vol. 195, edited by F. Binder, L. A. Correa, C. Gogolin, J. Anders, and G. Adesso (Springer International Publishing, Cham, 2018) pp. 363-393.

[53] M. H. Mohammady, A. Auffèves, and J. Anders, Commun. Phys. 3, 89 (2020).

[54] C. Menczel, Paul Flindt and K. Brandner, Phys. Rev. Research 2, 033449 (2020).

[55] K. W. Murch, S. J. Weber, C. Macklin, and I. Siddiqi, Nature 502, 211 (2013).

[56] J. P. Pekola, P. Solinas, A. Shnirman, and D. V. Averin, N. J. Phys 15, 115006 (2013).

[57] M. Naghiloo, D. Tan, P. M. Harrington, J. J. Alonso, E. Lutz, A. Romito, and K. W. Murch, Phys. Rev. Lett. 124, 110604 (2020).

[58] P. Talkner, E. Lutz, and P. Hänggi, Phys. Rev. E 75, 050102 (2007)

[59] M. Esposito, U. Harbola, and S. Mukamel, Rev. Mod. Phys. 81, 1665 (2009).

[60] H. J. D. Miller, M. H. Mohammady, M. Perarnau-Llobet, and G. Guarnieri, Phys. Rev. E 103, 052138 (2021).

[61] Denes Petz and S. Szabo, Int. J. Math. , 1 (2009).

[62] F. Hansen, Proc. Natl. Acad. Sci. USA 105, 9909 (2008).

[63] E. P. Wigner and M. M. Yanase, J. Phys. Chem. 15, 1084 (1963).

[64] S. Luo, Phys. Rev. A 73, 022324 (2006).

\section{A. Entropy production and work as a scalar product}

Consider a system with a separable Hilbert space $\mathcal{H}$, with $\mathcal{B}(\mathcal{H}) \supset \mathcal{B}_{S}(\mathcal{H})$ denoting the algebra of bounded operators, and real vector space of self-adjoint operators on $\mathcal{H}$, respectively, and $\mathcal{T}(\mathcal{H}) \subseteq \mathcal{B}(\mathcal{H})$ the space of trace-class operators, i.e., the bounded operators for which the trace is well-defined, and finite. Note that for any $A \in \mathcal{B}(\mathcal{H})$ and $B \in \mathcal{T}(\mathcal{H})$, we have $A B, B A \in \mathcal{T}(\mathcal{H})$, while $\mathcal{T}(\mathcal{H})=\mathcal{B}(\mathcal{H})$ if $\mathcal{H}$ is finite-dimensional. The normal state space of $\mathcal{H}$ is thus defined as the space of positive, unit-trace operators $\mathcal{S}(\mathcal{H}) \subset \mathcal{T}(\mathcal{H})$.

Let us define a parameter process by the function $\lambda:[0, \tau] \ni t \mapsto \lambda(t):=\{T(t), \Lambda(t)\}$, where $T(t)$ is the temperature of the surrounding thermal bath, and $\Lambda(t)$ is the mechanical parameter of the system Hamiltonian, $H_{\Lambda(t)}$. Denoting the equilibrium state, for each $\lambda(t)$, as $\pi_{\lambda(t)}:=e^{-H_{\Lambda(t)} / T(t)} / \operatorname{Tr}\left(e^{-H_{\Lambda(t)} / T(t)}\right)$, we may define the "spectrum shift" $\delta: A_{\lambda(t)} \mapsto \delta A_{\lambda(t)}:=A_{\lambda(t)}-\operatorname{Tr}\left(A_{\lambda(t)} \pi_{\lambda(t)}\right) \mathbb{I}$, so that for any $A_{\lambda(t)}, \operatorname{Tr}\left(\delta A_{\lambda(t)} \pi_{\lambda(t)}\right)=0$. Therefore, let us introduce the space of self-adjoint operator valued functions with zero expectation value at equilibrium, $\delta A_{\lambda}:[0, \tau] \rightarrow \mathcal{B}_{S}(\mathcal{H})$, defined as the set

$$
\mathcal{B}_{S}(\mathcal{H}, \lambda):=\left\{\delta A_{\lambda}:[0, \tau] \ni t \mapsto \delta A_{\lambda(t)}\right\}
$$

Note that by construction, $\delta \mathbb{I}=0$, i.e., the identity $\mathbb{I}$ is never in the range of any $\delta A_{\lambda} \in \mathcal{B}_{S}(\mathcal{H}, \lambda)$. Moreover, we recall that the quantities considered in the main text, in terms of which we established the thermodynamic uncertainty relation, are of the form $\delta \dot{X}_{\lambda(t)}$, with $X_{\lambda(t)}:=(1 / T(t)) H_{\Lambda(t)}$, and $\delta \dot{H}_{\lambda(t)}$. Clearly, $\delta \dot{X}_{\lambda}, \delta \dot{H}_{\lambda} \in \mathcal{B}_{S}(\mathcal{H}, \lambda)$.

Now let us turn to the setup described in the main text, where the system evolves according to a Lindbladian $\mathscr{L}_{\lambda(t)}$, which in the Heisenberg picture is given by the dual $\mathscr{L}_{\lambda(t)}^{*}$, defined by the identity $\operatorname{Tr}\left(\mathscr{L}_{\lambda(t)}^{*}(A) B\right)=\operatorname{Tr}\left(A \mathscr{L}_{\lambda(t)}(B)\right)$ for 
all $A \in \mathcal{B}(\mathcal{H})$ and $B \in \mathcal{T}(\mathcal{H})$, taking the form

$$
\mathscr{L}_{\lambda(t)}^{*}(\cdot):=\mathscr{H}_{\Lambda(t)}(\cdot)+\sum_{x}\left(L_{x}(\lambda(t))^{\dagger}(\cdot) L_{x}(\lambda(t))-\frac{1}{2}\left\{L_{x}(\lambda(t))^{\dagger} L_{x}(\lambda(t)),(\cdot)\right\}\right) .
$$

Here, we define the superoperator $\mathscr{H}_{\Lambda(t)}(\cdot):=i\left[H_{\Lambda(t)},(\cdot)\right]$ which describes the system's Hamiltonian dynamics. We assume that the equilibrium state $\pi_{\lambda(t)}$ is the unique fixed point of the Quantum Markov Semigorup generated by $\mathscr{L}_{\lambda(t)}$; a state $\rho \in \mathcal{S}(\mathcal{H})$ is a fixed point of $e^{\theta \mathscr{L}_{\lambda(t)}}$ if and only if $\rho$ is in the kernel of $\mathscr{L}_{\lambda(t)}$, i.e., $\mathscr{L}_{\lambda(t)}(\rho)=0$. In other words, we demand that for any state $\rho \in \mathcal{S}(\mathcal{H}), \mathscr{L}_{\lambda(t)}(\rho)=0$ if and only if $\rho=\pi_{\lambda(t)}$.

Note that the fixed point $\pi_{\lambda(t)}$ is faithful; a state $\rho \in \mathcal{S}(\mathcal{H})$ is faithful if for any $A \in \mathcal{B}(\mathcal{H}), \operatorname{Tr}\left(A^{\dagger} A \rho\right)=0 \Longleftrightarrow A$. If $\mathcal{H}$ is finite-dimensional, a faithful state can be considered as one that has full rank, i.e., a state with no zero-eigenvalues. The faithfulness of a fixed point $\pi_{\lambda(t)}$ implies that, for any $s \in[0,1]$, we may define the $s$-dual of $\mathscr{L}_{\lambda(t)}^{*}$, denoted $\tilde{\mathscr{L}}_{\lambda(t)}^{(s)}$, as the solution to

$$
\operatorname{Tr}\left(\pi_{\lambda(t)}^{1-s} \tilde{\mathscr{L}}_{\lambda(t)}^{(s)}(A) \pi_{\lambda(t)}^{s} B\right)=\operatorname{Tr}\left(\pi_{\lambda(t)}^{1-s} A \pi_{\lambda(t)}^{s} \mathscr{L}_{\lambda(t)}^{*}(B)\right)
$$

for all $A, B \in \mathcal{B}(\mathcal{H})$. Recall from the main text that we only consider systems which obey the detailed balance condition. $\mathscr{L}_{\lambda(t)}^{*}$ obeys the $s$-detailed balance condition if $\tilde{\mathscr{L}}_{\lambda(t)}^{(s)}=\mathscr{L}_{\lambda(t)}^{*}-2 \mathscr{H}_{\lambda(t)}$. However, as shown by Fagnola et al [78] $\mathscr{L}_{\lambda(t)}^{*}$ obeys the 0-detailed balance condition if and only if $\mathscr{L}_{\lambda(t)}^{*}$ admits a privileged representation (Theorem 5.1), which in turn implies that the $s$-dual will be uniquely defined as $\tilde{\mathscr{L}}_{\lambda(t)}^{(s)}=\tilde{\mathscr{L}}_{\lambda(t)}$ for all $s$ (Propositions 7.1 and 8.1 ). As such, we shall simplify the notation and state that $\mathscr{L}_{\lambda(t)}^{*}$ obeys detailed balance if

$$
\tilde{\mathscr{L}}_{\lambda(t)}=\mathscr{L}_{\lambda(t)}^{*}-2 \mathscr{H}_{\lambda(t)}
$$

Moreover, since we always assume that the stationary state $\pi_{\lambda(t)}$ is of Gibbs form, if $\mathscr{L}_{\lambda(t)}^{*}$ obeys detailed balance, then it necessarily also obeys time-translation covariance (see more details in the accompanying article [60]), defined as

$$
\mathscr{H}_{\Lambda(t)} \circ \mathscr{L}_{\lambda(t)}^{*}=\mathscr{L}_{\lambda(t)}^{*} \circ \mathscr{H}_{\Lambda(t)}
$$

Now consider the symmetric bilinear forms $\langle\langle\cdot, \cdot\rangle\rangle_{\lambda}: \mathcal{B}_{S}(\mathcal{H}, \lambda) \times \mathcal{B}_{S}(\mathcal{H}, \lambda) \rightarrow \mathbb{R}$ and $\langle\langle\cdot, \cdot\rangle\rangle_{\lambda}^{\prime}: \mathcal{B}_{S}(\mathcal{H}, \lambda) \times \mathcal{B}_{S}(\mathcal{H}, \lambda) \rightarrow \mathbb{R}$, defined as

$$
\begin{aligned}
& \left\langle\left\langle\delta A_{\lambda}, \delta B_{\lambda}\right\rangle\right\rangle_{\lambda}:=\frac{1}{2 \tau} \int_{0}^{\tau} d t \int_{0}^{\infty} d \theta \operatorname{Tr}\left(\delta A_{\lambda(t)}(\theta) \mathbb{J}_{\lambda(t)}\left(\delta B_{\lambda(t)}(0)\right)+\delta B_{\lambda(t)}(\theta) \mathbb{J}_{\lambda(t)}\left(\delta A_{\lambda(t)}(0)\right)\right) \\
& \left\langle\left\langle\delta A_{\lambda}, \delta B_{\lambda}\right\rangle\right\rangle_{\lambda}^{\prime}:=\frac{1}{2 \tau} \int_{0}^{\tau} d t \int_{0}^{\infty} d \theta \operatorname{Tr}\left(\delta A_{\lambda(t)}(\theta) \mathbb{S}_{\lambda(t)}\left(\delta B_{\lambda(t)}(0)\right)+\delta B_{\lambda(t)}(\theta) \mathbb{S}_{\lambda(t)}\left(\delta A_{\lambda(t)}(0)\right)\right)
\end{aligned}
$$

Here, we define $\delta A_{\lambda(t)}(\theta):=e^{\theta \mathscr{L}_{\lambda(t)}^{*}}\left(\delta A_{\lambda(t)}\right)$ as the Heisenberg evolved operator $\delta A_{\lambda(t)}$, and we have introduced the logarithmic matrix mean

$$
\mathbb{J}_{\lambda(t)}(\cdot):=\int_{0}^{1} d s \pi_{\lambda(t)}^{s}(\cdot) \pi_{\lambda(t)}^{1-s}
$$

and the arithmetic matrix mean

$$
\mathbb{S}_{\lambda(t)}(\cdot):=\frac{1}{2}\left\{(\cdot), \pi_{\lambda(t)}\right\}
$$

We note that these matrix means satisfy the following properties:

$$
\mathbb{J}_{\lambda(t)}(A)^{\dagger}=\mathbb{J}_{\lambda(t)}\left(A^{\dagger}\right), \quad \mathbb{S}_{\lambda(t)}(A)^{\dagger}=\mathbb{S}_{\lambda(t)}\left(A^{\dagger}\right) \quad \forall A \in \mathcal{B}(\mathcal{H}),
$$

and

$$
\mathbb{J}_{\lambda(t)}^{*}=\mathbb{J}_{\lambda(t)}, \quad \quad \mathbb{S}_{\lambda(t)}^{*}=\mathbb{S}_{\lambda(t)}
$$

In other words, these matrix means are *-homomorphisms (Eq. (A9)), and are self-dual (Eq. (A10)). 
Theorem 1. Consider a Quantum Markov Semigorup, generated by the Lindbladian $\mathscr{L}_{\lambda(t)}^{*}$ defined in Eq. (A2), which satisfies the following properties: (i) there exists a faithful state $\pi_{\lambda(t)}$ such that $\mathscr{L}_{\lambda(t)}\left(\pi_{\lambda(t)}\right)=0$; (ii) $\mathscr{L}_{\lambda(t)}^{*}$ satisfies detailed balance and time-translation covariance; and (iii) for any $\rho \in \mathcal{S}(\mathcal{H})$, if $\mathscr{L}_{\lambda(t)}(\rho)=0$, then $\rho=\pi_{\lambda(t)}$. The bilinear forms (A6) thus define a pair of inner products satisfying

$$
\left\langle\left\langle\delta A_{\lambda}, \delta A_{\lambda}\right\rangle\right\rangle_{\lambda}^{\prime} \geq\left\langle\left\langle\delta A_{\lambda}, \delta A_{\lambda}\right\rangle\right\rangle_{\lambda} \geq 0
$$

for all $A_{\lambda} \in \mathcal{B}_{S}(\mathcal{H}, \lambda)$.

Proof. To simplify notation, we will drop $\delta$, and all subscripts related to $\lambda$ and $t$, i.e, $\delta A_{\lambda}=A, \delta A_{\lambda(t)}=A, H_{\Lambda(t)}=H$, $\pi_{\lambda(t)}=\pi, \mathbb{J}_{\lambda(t)}=\mathbb{J}, \mathbb{S}_{\lambda(t)}=\mathbb{S}$ etc. We begin by introducing the inner product $\langle\cdot, \cdot\rangle: \mathcal{B}_{S}(\mathcal{H}) \times \mathcal{B}_{S}(\mathcal{H}) \rightarrow \mathbb{R}$, defined as

$$
\langle A, B\rangle:=\operatorname{Tr}(A \mathbb{J}(B)), \quad \mathbb{J}(\cdot):=\int_{0}^{1} d s \pi^{s}(\cdot) \pi^{1-s} .
$$

Note that Eq. (A9) and cyclicity of the trace ensures that $\langle A, B\rangle=\langle A, B\rangle^{*}$. Moreover, given that $B$ is a bounded operator, while $\pi$ is trace-class, it follows that $\mathbb{J}(B)$ is also trace-class; this implies that $|\langle A, B\rangle|\langle\infty$. To show that $\langle\cdot, \cdot\rangle$ is an inner product, we note that it satisfies the following properties: (i) linearity in the first argument, $\langle\alpha A+B, C\rangle=\alpha\langle A, C\rangle+\langle B, C\rangle$ where $\alpha \in \mathbb{R}$; (ii) symmetry, $\langle A, B\rangle=\langle B, A\rangle$; and (iii) positive definiteness $\langle A, A\rangle \geq 0$. Property (i) is trivial. To show property (ii), we use cyclicity of the trace and Eq. (A10) to show that

$$
\langle A, B\rangle=\operatorname{Tr}(A \mathbb{J}(B))=\operatorname{Tr}(\mathbb{J}(A) B)=\langle B, A\rangle .
$$

Finally, to show property (iii), note that for any $s \in[0,1], \operatorname{Tr}\left(A \pi^{s} A \pi^{1-s}\right)=\operatorname{Tr}\left(M^{\dagger} M\right) \geqslant 0$, where $M=\pi^{s / 2} A \pi^{(1-s) / 2}$.

Now observe that the nested commutator $\mathcal{C}_{m}$, defined as $\mathcal{C}_{0}(A)=A$ for all $A \in \mathcal{B}(\mathcal{H})$ and $\mathcal{C}_{m+1}(\cdot)=\left[H, \mathcal{C}_{m}(\cdot)\right]$, where $H \in \mathcal{B}_{S}(\mathcal{H})$ is the Hamiltonian, satisfies

$$
\operatorname{Tr}\left(A \mathcal{C}_{m}(B)\right)=(-1)^{m} \operatorname{Tr}\left(\mathcal{C}_{m}(A) B\right)
$$

for all $A, B \in \mathcal{B}_{S}(\mathcal{H})$ and $m \in \mathbb{Z}$ (assuming that at least one of $A, B$ is also trace-class). Furthermore, using $[\pi, H]=0$ it is straightforward to verify the commutation relations

$$
\mathbb{J} \circ \mathcal{C}_{m}=\mathcal{C}_{m} \circ \mathbb{J}, \quad \forall m \in \mathbb{Z} .
$$

We thus find for any $A, B \in \mathcal{B}_{S}(\mathcal{H})$ the following:

$$
\begin{aligned}
\left\langle A, \mathcal{C}_{m}(B)\right\rangle & =\operatorname{Tr}\left(A \mathbb{J} \circ \mathcal{C}_{m}(B)\right), \\
& =\operatorname{Tr}\left(\mathbb{J}(A) \mathcal{C}_{m}(B)\right), \\
& =(-1)^{m} \operatorname{Tr}\left(\mathcal{C}_{m} \circ \mathbb{J}(A) B\right), \\
& =(-1)^{m} \operatorname{Tr}\left(\mathbb{J} \circ \mathcal{C}_{m}(A) B\right), \\
& =(-1)^{m}\left\langle B, \mathcal{C}_{m}(A)\right\rangle .
\end{aligned}
$$

Here: we use Eq. (A10) in the second line; in the third line, we use Eq. (A9) which implies that for all $A \in \mathcal{B}_{S}(\mathcal{H})$, the trace-class operator $\mathbb{J}(A)$ is also self-adjoint, justifying the use of Eq. (A14); and we use Eq. (A15) in the fourth line.

Given the assumption that $\mathscr{L}$ has a faithful state in its kernel, i.e., $\pi$, we may use the definition of the $s$-dual in Eq. (A3). Moreover, given the assumption that $\mathscr{L}^{*}$ obeys detailed balance Eq. (A4) and time-translation covariance Eq. (A5), we may thus write

$$
\begin{aligned}
\langle A(\theta), B\rangle & =\int_{0}^{1} d s \operatorname{Tr}\left(A(\theta) \pi^{s} B \pi^{1-s}\right), \\
& =\int_{0}^{1} d s \operatorname{Tr}\left(e^{\theta \mathscr{L}^{*}}(A) \pi^{s} B \pi^{1-s}\right), \\
& =\int_{0}^{1} d s \operatorname{Tr}\left(e^{\frac{\theta}{2} \mathscr{L}^{*}}(A) \pi^{s} e^{\frac{\theta}{2} \tilde{\mathscr{L}}}(B) \pi^{1-s}\right), \\
& =\int_{0}^{1} d s \operatorname{Tr}\left(e^{\frac{\theta}{2} \mathscr{L}^{*}}(A) \pi^{s} e^{\frac{\theta}{2}\left(\mathscr{L}^{*}-2 \mathscr{H}\right)}(B) \pi^{1-s}\right), \\
& =\int_{0}^{1} d s \operatorname{Tr}\left(A(\theta / 2) \pi^{s} e^{-\theta \mathscr{H}}(B(\theta / 2)) \pi^{1-s}\right), \\
& =\left\langle A(\theta / 2), e^{-\theta \mathscr{H}}(B(\theta / 2))\right\rangle=\sum_{m=0}^{\infty} \frac{(-i \theta)^{m}}{m !}\left\langle A(\theta / 2), \mathcal{C}_{m}(B(\theta / 2))\right\rangle,
\end{aligned}
$$


where we use the exponential series $e^{-\theta \mathscr{H}}=\sum_{m=0}^{\infty} \frac{(-i \theta)^{m}}{m !} \mathcal{C}_{m}$. Consequently, by Eq. (A6) we have

$$
\begin{aligned}
\langle\langle A, B\rangle\rangle & :=\frac{1}{2 \tau} \int_{0}^{\tau} d t \int_{0}^{\infty} d \theta(\langle A(\theta), B(0)\rangle+\langle B(\theta), A(0)\rangle) \\
& =\frac{1}{2 \tau} \int_{0}^{\tau} d t \int_{0}^{\infty} d \theta \sum_{m=0}^{\infty} \frac{(-i \theta)^{m}}{m !}\left(1+(-1)^{m}\right)\left\langle A(\theta / 2), \mathcal{C}_{m}(B(\theta / 2))\right\rangle \\
& =\frac{1}{\tau} \int_{0}^{\tau} d t \int_{0}^{\infty} d \theta \sum_{n=0}^{\infty} \frac{(i \theta)^{2 n}}{(2 n) !}\left\langle A(\theta / 2), \mathcal{C}_{2 n}(B(\theta / 2))\right\rangle \\
& =\frac{1}{\tau} \int_{0}^{\tau} d t \int_{0}^{\infty} d \theta \sum_{n=0}^{\infty} \frac{(i \theta)^{2 n}}{(2 n) !} \operatorname{Tr}\left(A(\theta / 2) \mathbb{J} \circ \mathcal{C}_{2 n}(B(\theta / 2))\right) \\
& =\frac{1}{\tau} \int_{0}^{\tau} d t \int_{0}^{\infty} d \theta \sum_{n=0}^{\infty} \frac{(i \theta)^{2 n}}{(2 n) !} \operatorname{Tr}\left(A(\theta / 2) \mathcal{C}_{n} \circ \mathbb{J} \circ \mathcal{C}_{n}(B(\theta / 2))\right) \\
& =\frac{1}{\tau} \int_{0}^{\tau} d t \int_{0}^{\infty} d \theta \sum_{n=0}^{\infty} \frac{(-1)^{n}(i \theta)^{2 n}}{(2 n) !}\left\langle\mathcal{C}_{n}(A(\theta / 2)), \mathcal{C}_{n}(B(\theta / 2))\right\rangle, \\
& =\frac{1}{\tau} \int_{0}^{\tau} d t \int_{0}^{\infty} d \theta \sum_{n=0}^{\infty} \frac{\theta^{2 n}}{(2 n) !}\left\langle\mathcal{C}_{n}(A(\theta / 2)), \mathcal{C}_{n}(B(\theta / 2))\right\rangle
\end{aligned}
$$

where we used $(\mathrm{A} 16)$ in the second and penultimate line. Since this equation is a positive weighted integral of the inner product $\langle\cdot, \cdot\rangle: \mathcal{B}_{S}(\mathcal{H}) \times \mathcal{B}_{S}(\mathcal{H}) \rightarrow \mathbb{R}$, this guarantees that the bilinear form $\langle\langle\cdot, \cdot\rangle\rangle: \mathcal{B}_{S}(\mathcal{H}, \lambda) \times \mathcal{B}_{S}(\mathcal{H}, \lambda) \rightarrow \mathbb{R}$ is also an inner product, i.e., it satisfies the properties: (i) linearity in the first argument $\langle\langle\alpha A+B, C\rangle\rangle=\alpha\langle\langle A, C\rangle\rangle+\langle\langle B, C\rangle\rangle$, where $\alpha \in \mathbb{R}$; (ii) symmetry $\langle\langle A, B\rangle\rangle=\langle\langle B, A\rangle\rangle$; and (iii) positive definiteness $\langle\langle A, A\rangle\rangle \geq 0$.

We can apply the exact same arguments for the alternative inner product $\langle\cdot, \cdot\rangle^{\prime}: \mathcal{B}_{S}(\mathcal{H}) \times \mathcal{B}_{S}(\mathcal{H}) \rightarrow \mathbb{R}$, defined as

$$
\langle A, B\rangle^{\prime}:=\operatorname{Tr}(A \mathbb{S}(B)), \quad \mathbb{S}(\cdot):=\frac{1}{2}\{(\cdot), \pi\}
$$

in which case we find

$$
\langle\langle A, B\rangle\rangle^{\prime}:=\frac{1}{2 \tau} \int_{0}^{\tau} d t \int_{0}^{\infty} d \theta\left(\langle A(\theta), B(0)\rangle^{\prime}+\langle B(\theta), A(0)\rangle^{\prime}\right)=\frac{1}{\tau} \int_{0}^{\tau} d t \int_{0}^{\infty} d \theta \sum_{n=0}^{\infty} \frac{\theta^{2 n}}{(2 n) !}\left\langle\mathcal{C}_{n}(A(\theta / 2)), \mathcal{C}_{n}(B(\theta / 2))\right\rangle^{\prime}
$$

An important remark is worth being made regarding the convergence of the inner products $\langle\langle\cdot, \cdot\rangle\rangle$ and $\langle\langle\cdot, \cdot\rangle\rangle^{\prime}$. We note that thus far, we have only used the fact that $\mathscr{L}$ has a faithful state $\pi$ in its kernel, and that $\mathscr{L}^{*}$ obeys detailed balance and time-translation covariance. The final assumption in the theorem was that $\pi$ is the unique state in the kernel of $\mathscr{L}$, which is precisely what ensures that the inner products is bounded, and hence meaningful. To show this, let us define the following superoperator:

$$
\mathscr{L}^{+}(\cdot):=-\int_{0}^{\infty} d \theta e^{\theta \mathscr{L}}((\cdot)-\pi \operatorname{Tr}(\cdot)) .
$$

It can be shown that Eq. (A21), also known as Drazin inverse of $\mathscr{L}$, satisfies the following properties [43, 60, 79]:

(i) $\operatorname{Tr}\left(\mathscr{L}^{+}(X)\right)=0$ for all $X \in \mathcal{T}(\mathcal{H})$.

(ii) $\mathscr{L} \mathscr{L}^{+}(X)=\mathscr{L}^{+} \mathscr{L}(X)=X-\operatorname{Tr}(X) \pi$ for all $X \in \mathcal{T}(\mathcal{H})$.

(iii) $\mathscr{L}^{+}(\pi)=0$.

The Drazin inverse corresponds to the minus- 1 power of the Lindblad generator. For the sake of simplicity, let us assume that $\mathscr{L}$ is "fully diagonalizable", i.e. $\mathscr{L}=S \Omega S^{-1}$, with the columns (rows) of the invertible matrix $S\left(S^{-1}\right)$ the right (left) eigenvectors, and $\Omega$ the diagonal matrix of complex eigenvalues, which (without loss of generality and upon reshuffling) we can take to be ordered such that $\Omega_{0}=0$ and $\left|\Omega_{k}\right|<\left|\Omega_{k+1}\right|$, with $0<\left|\Omega_{k}\right|<\infty \forall k>0$. The fact that $\mathscr{L}$ has a unique state $\pi$ in its kernel is reflected by the existence of only one eigenvalue $\Omega_{k}$ such that $\left|\Omega_{k}\right|=0$, namely, $\Omega_{0}$. The spectral decomposition of the Drazin inverse can be shown to be simply given by $\mathscr{L}^{+}=S \tilde{\Omega}^{-1}$, where $\tilde{\Omega}_{k}=\Omega_{k}^{-1}$, 
i.e. it corresponds to the inverse spectrum of the Lindbladian excluding the zero eigenvalue. In other words, $\Omega_{0}=0$ continues to be in the spectrum $\tilde{\Omega}$, so that $\pi$ continues to be in the kernel of $\mathscr{L}^{+}$, see property (iii) above. Since for all $k>0,\left|\Omega_{k+1}\right|>\left|\Omega_{k}\right|>0$, then $\left|\tilde{\Omega}_{k+1}\right|<\left|\tilde{\Omega}_{k}\right|<\infty$; the Drazin inverse will have a bounded spectrum. Consequently, for any operator $X \in \mathcal{T}(\mathcal{H})$, then $\mathscr{L}^{+}(X) \in \mathcal{T}(\mathcal{H})$. Indeed, this condition is implicit in properties (i) and (ii). Notably, the boundedness of the Drazin inverse can be shown also when the Linblad generator is only "Jordan-diagonalizable" by exploiting the closed analytical expression of its spectrum (see Appendices A to G of [80]).

In order to see how this allows us to achieve the goal of determining the convergence of the bilinear form Eq. (A18), the last step is to evaluate the following:

$$
\begin{aligned}
\int_{0}^{\infty} d \theta\langle A(\theta), B\rangle & =\int_{0}^{\infty} d \theta \operatorname{Tr}(A(\theta) \mathbb{J}(B))=\int_{0}^{\infty} d \theta \operatorname{Tr}\left(e^{\theta \mathscr{L}^{*}}(A) \mathbb{J}(B)\right), \\
& =\int_{0}^{\infty} d \theta \operatorname{Tr}\left(A e^{\theta \mathscr{L}}(\mathbb{J}(B))\right), \\
& =-\operatorname{Tr}\left(A \mathscr{L}^{+}(\mathbb{J}(B))\right)+\int_{0}^{\infty} d \theta \operatorname{Tr}\left(A e^{\theta \mathscr{L}}(\pi)\right) \operatorname{Tr}(\mathbb{J}(B)), \\
& =-\operatorname{Tr}\left(A \mathscr{L}^{+}(\mathbb{J}(B))\right),
\end{aligned}
$$

where we used the definition of the dual in the second line (see above Eq. (A2)), the definition of the Drazin inverse Eq. (A21) with the argument being the trace-class operator $\mathbb{J}(B)$ in the third line, and finally where $\operatorname{Tr}(\mathbb{J}(B))=\operatorname{Tr}(\pi B)=0$ is used in the fourth line (this stems directly from the fact that we consider only self-adjoint operator valued functions with a zero expectation value at equilibrium, see Eq. (A1)). Due to the arguments made above, since $A, B \in \mathcal{B}_{S}(\mathcal{H})$, and $\mathscr{L}^{+}(\mathbb{J}(B)) \in \mathcal{T}(\mathcal{H})$, therefore $A \mathscr{L}^{+}(\mathbb{J}(B)) \in \mathcal{T}(\mathcal{H})$, and hence the trace in the final line of Eq. (A22) is a finite quantity. Since the bilinear form Eq. (A18) consists of a finite integral, over the total driving time $\tau$, of two terms such as Eq. (A22), it therefore converges to a finite quantity. The exact same arguments can also be used to show the boundedness of Eq. (A20).

Finally, we note that the Kubo-Ando inequality implies that the arithmetic matrix mean is maximal amongst the family of means [81]. In particular, one has $\langle A, A\rangle^{\prime} \geq\langle A, A\rangle$. Comparing (A18) and (A20) then implies the inequalities

$$
\langle\langle A, A\rangle\rangle^{\prime} \geq\langle\langle A, A\rangle\rangle \geq 0 \text {. }
$$

As stated in the main text, the average entropy production, as well as the average power and its fluctuations, can be expressed in terms of the inner product $\langle\langle\cdot, \cdot\rangle\rangle_{\lambda}$. In particular, the fluctuating power is

$$
\Delta P_{w}=2\left(\Delta \mathcal{I}_{w}+\left\langle\left\langle\delta \dot{H}_{\lambda}, \delta \dot{H}_{\lambda}\right\rangle\right\rangle_{\lambda}\right) \equiv 2\left\langle\left\langle\delta \dot{H}_{\lambda}, \delta \dot{H}_{\lambda}\right\rangle\right\rangle_{\lambda}^{\prime},
$$

where the quantum correction term obeys the identity

$$
\Delta \mathcal{I}_{w}:=\frac{1}{\tau} \int_{0}^{\tau} d t \int_{0}^{\infty} d \theta \mathcal{I}_{\lambda(t)}\left(\dot{H}_{\Lambda(t)}(\theta), \dot{H}_{\Lambda(t)}(0)\right) \equiv\left\langle\left\langle\delta \dot{H}_{\lambda}, \delta \dot{H}_{\lambda}\right\rangle\right\rangle_{\lambda}^{\prime}-\left\langle\left\langle\delta \dot{H}_{\lambda}, \delta \dot{H}_{\lambda}\right\rangle\right\rangle_{\lambda} .
$$

As a consequence of Theorem 1 , the quantum correction term $\Delta \mathcal{I}_{w}$ is non-negative, and obeys the inequality

$$
\Delta P_{w} \geq 2 \Delta \mathcal{I}_{w} \geq 0 .
$$

\section{B. Example: a single ion heat engine}

We shall now turn to the example of a single ion heat engine, which can be modelled as a harmonic oscillator. To be sure, the observables of interest for a harmonic oscillator, such as the Hamiltonian, are unbounded, whereas our results thus far have been framed in terms of bounded operators. Notwithstanding, the model we consider admits a Master equation obeying detail balanced, and thus falls within the domain of applicability of our main results [82]. The Hamiltonian of the harmonic oscillator, with frequency $\omega$, takes the form

$$
\begin{aligned}
& H_{\omega}=\omega\left(a_{\omega}^{\dagger} a_{\omega}+\frac{1}{2}\right), \\
& x^{2}=\frac{1}{2 \omega}\left(\left(a_{\omega}^{\dagger}\right)^{2}+a_{\omega}^{2}+2 a_{\omega}^{\dagger} a_{\omega}+1\right), \\
& p^{2}=\frac{\omega}{2}\left(-\left(a_{\omega}^{\dagger}\right)^{2}-a_{\omega}^{2}+2 a_{\omega}^{\dagger} a_{\omega}+1\right) .
\end{aligned}
$$


For a fixed $\lambda=(T, \omega)$, the Lindblad superoperator governing the evolution of the harmonic oscillator, in the Heisenberg picture, is given as

$$
\mathscr{L}_{\lambda}^{*}(\cdot)=i \omega\left[a_{\omega}^{\dagger} a_{\omega},(\cdot)\right]+\Gamma\left(N_{\beta}+1\right) \tilde{\mathcal{D}}_{a_{\omega}}[(\cdot)]+\Gamma \tilde{\mathcal{D}}_{a_{\omega}^{\dagger}}[(\cdot)]
$$

with

$$
\tilde{\mathcal{D}}_{X}[\rho]=X^{\dagger} \rho X-\frac{1}{2}\left\{X^{\dagger} X, \rho\right\}
$$

We wish to compute:

$$
\begin{aligned}
& \langle\dot{\sigma}\rangle=\left\langle\left\langle\delta \dot{X}_{\lambda}, \delta \dot{X}_{\lambda}\right\rangle\right\rangle_{\lambda}, \\
& P_{w}=P_{\mathcal{W}}-\left\langle\left\langle\delta \dot{H}_{\lambda}, \delta \dot{X}_{\lambda}\right\rangle\right\rangle_{\lambda}, \\
& \Delta P_{w}=2\left\langle\left\langle\delta \dot{H}_{\lambda}, \delta \dot{H}_{\lambda}\right\rangle\right\rangle_{\lambda}^{\prime}, \\
& \Delta I_{w}=\left\langle\left\langle\delta \dot{H}_{\lambda}, \delta \dot{H}_{\lambda}\right\rangle\right\rangle_{\lambda}^{\prime}-\left\langle\left\langle\delta \dot{H}_{\lambda}, \delta \dot{H}_{\lambda}\right\rangle\right\rangle_{\lambda} .
\end{aligned}
$$

A lengthy calculation yields (see more details in the accompanying article [60]):

$$
\begin{aligned}
P_{w} & =P_{\mathcal{W}}-\frac{1}{\tau} \int_{0}^{\tau} d t \frac{\dot{\omega} e^{\beta \omega}\left(\omega\left(\Gamma^{2}+4 \omega^{2}\right)(\dot{\beta} \omega+\beta \dot{\omega})+\Gamma^{2} \dot{\omega} \sinh (\beta \omega)\right)}{\Gamma \omega\left(e^{\beta \omega}-1\right)^{2}\left(\Gamma^{2}+4 \omega^{2}\right)} \\
\Delta P_{w} & =\frac{1}{\tau} \int_{0}^{\tau} d t \frac{2 \dot{\omega}^{2} e^{\beta \omega}\left(\Gamma^{2}+4 \omega^{2}+\Gamma^{2} \cosh (\beta \omega)\right)}{\left(e^{\beta \omega}-1\right)^{2}\left(\Gamma^{3}+4 \Gamma \omega^{2}\right)} \\
\langle\dot{\sigma}\rangle & =\frac{1}{\tau} \int_{0}^{\tau} d t \frac{e^{\beta \omega}\left(\beta \Gamma^{2} \dot{\omega}^{2} \sinh (\beta \omega)+\omega\left(\Gamma^{2}+4 \omega^{2}\right)(\dot{\beta} \omega+\beta \dot{\omega})^{2}\right)}{\Gamma \omega\left(e^{\beta \omega}-1\right)^{2}\left(\Gamma^{2}+4 \omega^{2}\right)} .
\end{aligned}
$$

Noting that $\operatorname{Tr}\left(\dot{H}_{\omega} \pi_{\lambda}\right)=\dot{\omega} \partial_{\omega} F_{\lambda}$, while $F_{\lambda}=-\beta^{-1} \ln \left(\frac{e^{\beta \omega}}{e^{\beta \omega}-1}\right)$, the adiabatic power is given by:

$$
P_{\mathcal{W}}=-\frac{1}{\tau} \int_{0}^{\tau} d t \operatorname{Tr}\left(\dot{H}_{\omega} \pi_{\lambda}\right)=-\frac{1}{\tau} \int_{0}^{\tau} d t \dot{\omega} \partial_{\omega} F_{\lambda}=-\frac{1}{\tau} \int_{0}^{\tau} d t \frac{\dot{\omega}}{e^{\beta \omega}-1}
$$

Therefore, we may compute the efficiency using the above expressions as

$$
\eta=\frac{\eta_{C} P_{w}}{T_{c}\langle\dot{\sigma}\rangle+P_{w}}
$$

To obtain the efficiency bound $\eta^{Q}$, we must now compute the quantum correction

$$
\Delta \mathcal{I}_{w}=\frac{1}{\tau} \int_{0}^{\tau} d t \int_{0}^{\infty} d \theta \operatorname{Tr}\left(\delta \dot{H}_{\lambda}(\theta)\left(\mathbb{S}_{\lambda}-\mathbb{J}_{\lambda}\right)\left(\delta \dot{H}_{\lambda}(0)\right)\right)
$$

which reduces to

$$
\Delta \mathcal{I}_{w}=\frac{1}{\tau} \int_{0}^{\tau} d t \frac{\dot{\omega}^{2} \Gamma\left(e^{2 \beta \omega}-1\right)(\beta \omega \operatorname{coth}(\beta \omega)-1)}{2 \beta \omega\left(e^{\beta \omega}-1\right)^{2}\left(\Gamma^{2}+4 \omega^{2}\right)} \geqslant 0,
$$

with positivity guaranteed by the positivity of the integrand for all $t$.

Finally, to ensure a protocol that is both cyclic, $\lambda(t)=\lambda(\tau)$, and satisfies the slow-driving condition $\dot{\lambda}(0)=\dot{\lambda}(\tau)=\{0,0\}$, we shall use the following parameter protocol, given in the main text:

$$
\begin{aligned}
\omega(t) & =\omega_{0}\left(1+\frac{1}{2} \sin \left(\frac{2 \pi t}{\tau}\right)+\frac{1}{4} \sin \left(\frac{4 \pi t}{\tau}+\pi\right)\right), \\
T(t) & =\frac{T_{c} T_{h}}{T_{h}+\left(T_{c}-T_{h}\right) \sin ^{2}\left(\frac{\pi t}{\tau}\right)} .
\end{aligned}
$$

Note that substituting these functions into the above integrals cannot be computed analytically, so we proceed by numerical integration. The results are presented in the figures given in the main text. 